[0212-7199 (2004) 21: 1; pp 12-16] ANALES DE MEDICINA INTERNA Copyright $($ C 2004 ARAN EDICIONES, S.L.

An. MED. InTERnA (Madrid) Vol. $21, \mathrm{~N}^{\circ} 1$, pp. $12-16,2004$

\title{
Tomografía por emisión de positrones mediante PET-18FDG en lesiones pulmonares radiológicamente indeterminadas
}

\author{
G. RUIZ-HERNÁNDEZ ${ }^{1,2}$, R. DE JUAN ${ }^{2}$, A. SAMANES $S^{1,2}$, H. VEREA ${ }^{3}$, J. M. PEÑAS ${ }^{4}$, \\ A. VERES ${ }^{5} ;$ L. LAPEÑA ${ }^{1,2}$, R. MONTZ ${ }^{2}$, J. L. CARRERAS ${ }^{1}$

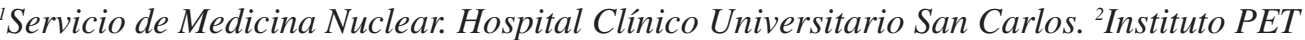 \\ Dr. Carreras. Madrid. Servicio de Neumología. ${ }^{3}$ Hospital Juan Canalejo. A Coruña. \\ ${ }^{4}$ Hospital Virgen de la Luz. Cuenca. ${ }^{5}$ Complexo Hospitalario Xeral-Calde. Lugo
}

POSITRON EMISSION TOMOGRAPHY WITH 18FDG-PET IN THE RADIOLOGICALLY INDETERMINATED PULMONARY LESIONS

\section{RESUMEN}

Objetivo: La Tomografía por Emisión de Positrones con 18-fluoro-2desoxi-D-glucosa (PET-FDG) es una técnica no-invasiva, que nos ofrece la posibilidad de caracterizar la benignidad o malignidad de las lesiones pulmonares (NPS) radiológicamente indeterminadas, con un elevado valor predictivo negativo y positivo. Observando las publicaciones indexadas podemos apreciar que son escasas las realizadas con las posibilidades diagnósticas de PET-FDG. Por eso, nuestro objetivo es analizar la exactitud diagnóstica (ED), de la PET en nuestra experiencia con 67 pacientes con lesiones nodulares radiológicamente indeterminadas.

Material y método: retrospectivamente, se ha valorado la aportación diagnóstica de la PET, de forma global (mediante análisis visual y semicuantitativo) y parcial (considerando únicamente el valor del SUV), en 67 pacientes con NPS radiológicamente indeterminado, efectuando la comprobación diagnóstica por anatomía patológica o seguimiento clínico- radiológico de duración superior a un año.

Resultados: Globalmente, la PET-FDG obtuvo una sensibilidad (S) del $92 \%$, una especificidad (E) del $86,6 \%$, un valor predictivo positivo (VPP) del $89.4 \%$, un valor predictivo negativo (VPN) del $89,6 \%$ y una exactitud diagnóstica (ED) del $89 \%$. Los mejores resultados se obtuvieron para un SUV (Standardized Uptake Value) igual o superior a 2,5, con una S de 92\%, E de 90\%, VPP 92\%, VPN 90\% y ED $91 \%$.

Conclusiones: Podemos caracterizar la mayoría de las lesiones pulmonares indeterminadas mediante la PET-FDG. El uso adicional del SUV posibilita aumentar el valor predictivo positivo y la especificidad de la técnica PET.

PALABRAS CLAVE: Tomografía por Emisión de Positrones (PETFDG). Lesión nodular pulmonar radiológicamente indeterminada.

\section{ABSTRACT}

Objective: Fluorine-18 deoxyglucose Positron Emission Tomography (FDG-PET) is a non-invasive technique that offers the possibility to define if the radiologically indeterminated pulmonary lesions are benign or malignant with high positive and negative predictive values. Considering the indexed literature we can observe that there are few original studies performed with the diagnostic possibilities of our means. For this reason, our main objective is to evalue the diagnostic accuracy of positron emission tomography in sixty-seven radiologically indeterminated pulmonary nodular lesions

Material and method: Restrospectively, we evaluated the diagnostic ability of FDG-PET globally (by means of visual and semicuantitative analysis) and partially (only considering the Standardized Uptake Value $(S U V))$, in sixty-seven patients confirmed by pathology or clinical and radiological monitoring, in a time interval superior to one year.

Results: Globally, FDG-PET had a sensitivity (S) of $92 \%$, specificity $(S P)$ of $86.6 \%$, positive predictive value $(P P V)$ of $89.4 \%$, negative predictive value (NPV) of $89.6 \%$ and diagnostic accuracy (DA) of $89 \%$. The best results were obtained for an SUV equal or superior to 2.5. With this value, the clinical efficacy parameters were: S 0.92, SP 0.90, PPV 0.92, NPV 0.90 and DA 0.91.

Conclusions: We can characterize most of the radiologically indeterminated pulmonary lesions by FDG-PET. The additional use of SUV facilitates an increase in the positive predictive value and specificity of FDG-PET.

KEY WORDS: Positron Emission Tomography (PET). Radiologically indeterminated pulmonary lesions.

Ruíz-Hernández G, de Juan R, Samanes A, Verea H, Peñas JM, Veres A, Lapeña L, Montz R, Carreras JL. Tomografía por Emisión de Positrones (PET-FDG). Lesión nodular pulmonar radiológicamente indeterminada. An Med Interna (Madrid) 2004; 21 : 12-16.

\section{INTRODUCCIÓN}

En la práctica asistencial diaria es frecuente encontrarse con una lesión pulmonar focal. El diagnóstico de estas lesiones debe ser lo más cuidadoso y eficiente para facilitar una resección tumoral lo más precoz posible de las lesiones malignas. El manejo asistencial incluye los métodos diagnósticos de imagen, la citología del esputo y la broncoscopia si se trata de lesiones centrales y de la punción-biopsia transtorácica si la lesión es periférica (1).

Trabajo aceptado: 04 de julio de 2003 
Los nódulos solitarios pulmonares suelen aparecer en una de cada quinientas radiografías de tórax practicadas. De las lesiones benignas, la mayoría son granulomas infecciosos $(80 \%)$ y hamartomas $(10 \%)$ y tumores benignos y granulomas no infecciosos (10\%). La prevalencia de malignidad varía entre un 10 a un $68 \%$, según los datos de la literatura (2).

Actualmente las pruebas de imagen más utilizadas en el diagnóstico de lesiones pulmonares, son la radiografía de tórax (3) y la tomografía axial computarizada (TAC) convencional (4) o de alta resolución $(5,6)$. Cuando estas técnicas no señalan la benignidad o malignidad del nódulo, es decir, son "radiológicamente indeterminadas", se recurre a la broncoscopia y /o PAAF transparietal (si son factibles). Si estas pruebas no son concluyentes o no se aconseja su realización, podemos efectuar una "Tomografía por Emisión de Positrones con 18-fluoro-2-desoxi-D-glucosa" (PET-FDG). La PET-FDG nos aportará información visual y semicuantitativa (mediante obtención del SUV ó Standardized Uptake Value) del consumo de glucosa de la lesión, colaborando así en la diferenciación de benignidad-malignidad del nódulo y ayudando en el manejo terapéutico de los pacientes (7-23).

Pese a los numerosos estudios publicados en la literatura, pocos se han desarrollado con PET-FDG en nuestro medio. Nuestro objetivo es analizar la exactitud diagnóstica (ED), sensibilidad (S), especificidad (E) y valor predictivo positivo (VPP) y negativo (VPN), que nos ofrece la técnica PET en 67 pacientes con lesiones nodulares radiológicamente indeterminadas.

\section{MATERIAL Y MÉTODOS}

Pacientes: se han incluido 67 pacientes con lesiones solitarias, radiológicamente indeterminadas y de origen desconocido, con los criterios propuestos por la AETS (23). En todos los casos se trataba de enfermos con lesiones pulmonares únicas, radiológicamente indeterminadas y con citología, broncoscopia o PAAF no concluyentes o no realizables por las características de los pacientes.

Se trataba de 51 varones y 16 mujeres, con edades comprendidas entre 23 y 78 años (Media de 60,5 años). Cincuenta y tres de los 67 no tenían antecedente remoto de tumor maligno previo y 14 sí ( 2 melanomas, 3 neoplasias de mama, 3 de recto, 2 vesicales, 1 ampolla de Water, 2 de cabeza y cuello y 1 de origen desconocido). Sólamente 2 de los pacientes presentaban lesiones cuyo diámetro mayor superaba los $4 \mathrm{~cm}$, siendo este diámetro de $2,1 \pm 1,3 \mathrm{~cm}$.

Tipo de estudio: de forma retrospectiva se ha valorado la aportación diagnóstica de la PET de manera global (mediante análisis visual y semicuantitativo) y parcial (considerando únicamente el valor del SUV máximo). El SUV es un índice semicuantitativo que relaciona la concentración del radiotrazador en la lesión a estudio con la actividad inyectada y el peso corporal, utilizándose de forma habitual como valor adicional de la interpretación cualitatitiva ó visual. El nivel de corte más utilizado para discriminar lesiones benignas de malignas extracerebrales se define entre los valores de 2,5 a 3 (21).

El diagnóstico definitivo se realizó mediante: cirugía $\left(\mathrm{n}^{\circ}=\right.$ 47, -70,5\%-), seguimiento clínico-radiológico con RX o TAC cada 6 meses durante los dos primeros años $\left(n^{\circ}=15,-22 \%\right.$-), biopsia $\left(n^{\circ}=2,-3 \%-\right)$, lavado broncoalveolar $\left(n^{\circ}=1,-1,5 \%-\right)$ y citología de esputo $\left(n^{\circ}=2,-3 \%-\right)$.

\section{CARACTERÍSTICAS DE LA EXPLORACIÓN}

Metodología: se inyectó a cada paciente una dosis de FDG de $185 \mathrm{MBq}(5 \mathrm{mCi})$ en situación de reposo. La preparación consistió en un mínimo de seis horas de ayuno previo y administración oral de $10 \mathrm{mg}$ de diacepam inmediatamente antes de la inyección y toma de una muestra de sangre para determinar la glucemia.

Tras la inyección los enfermos permanecieron en reposo (decúbito supino y semioscuridad) durante un mínimo de 45 minutos, realizándose hidratación con 0,5 litro de agua por vía oral.

La adquisición del estudio se realizó con una Cámara de Positrones modelo "C-PET 250" de "ADAC-PHILIPS". La duración de la adquisición fue de 40 minutos, en seis secciones transversales de $25 \mathrm{~cm}$ de eje axial con solapamiento de $12,5 \mathrm{~cm}$, incluyendo la emisión y la transmisión (mediante fuente externa rotatoria de $137 \mathrm{Cs}$ ).

Las imágenes de cuerpo completo se reconstruyeron durante la adquisición mediante reconstrucción iterativa, efectuando corrección de atenuación. Se obtuvieron cortes transaxiales, coronales y sagitales, así como proyecciones volumétricas de cuerpo completo. Cada exploración se analizó visualmente por tres observadores y de modo semicuantitativo mediante la determinación en la lesión del valor máximo del SUV (Standardized Uptake Value). La interpretación de la PET fue ciega con respecto al diagnóstico final.

\section{RESULTADOS}

El diagnóstico definitivo fue de lesiones malignas en 37 pacientes y de origen benigno en 30 pacientes. En la evaluación (análisis visual y semicuantitativo) la PET-FDG tuvo un resultado positivo en 38 casos (4 falsos positivos), negativo en 29 (tres falsos negativos), (Tabla I) ofreciendo una sensibilidad (S) del 92\%, una especificidad (E) del 86,6\%, un valor predictivo positivo (VPP) del $89,4 \%$, un valor predictivo negativo (VPN) del 89,6\% y una exactitud diagnóstica (ED) del $89 \%$. Los cuatro resultados falsos positivos fueron secundarios a: 2 hamartomas, uno con $\mathrm{SUV}=2,8$, diámetro $=3 \mathrm{~cm}$, y $\mathrm{CD}=$ cirugía; (Fig. 1) y el segundo con $\mathrm{SUV}=2$, diámetro= $1,5 \mathrm{~cm}$, y $\mathrm{CD}=$ cirugía; tuberculosis $(\mathrm{SUV}=2,2 ;$ diámetro $=2$ $\mathrm{cm} ; \mathrm{CD}=$ citología de esputo) y neumonía atípica ( $\mathrm{SUV}=2,5$; diámetro $=1,5 \mathrm{~cm} ; \mathrm{CD}=$ cirugía $)$. Los tres resultados falsos negativos se obtuvieron en: adenocarcinoma de origen desconocido $(\mathrm{SUV}=1,9$; diámetro $=1,6 \mathrm{~cm})$, fibrohistiocitoma maligno (con ausencia de fijación de FDG y diámetro= 1,3 $\mathrm{cm}$ ), y en un tercer caso (sin captación de FDG, con tamaño de $2 \mathrm{~cm}$ ) sin confirmación histológica (enfermo se negó a cirugía), pero con incremento del tamaño del nódulo en el seguimiento clínico-radiológico de 14 meses.

\section{TABLA I}

\begin{tabular}{|c|c|c|}
\hline \multicolumn{3}{|c|}{ RESULTADOS DE LA PET-FDG } \\
\hline & Enfermos & Sanos \\
\hline $\begin{array}{l}\text { PET positiva (38) } \\
\text { PET negativa (29) }\end{array}$ & $\begin{array}{l}\mathrm{VP}(34) \\
\mathrm{FN}(3)\end{array}$ & $\begin{array}{l}\text { FP (4) } \\
\text { VN (26) }\end{array}$ \\
\hline
\end{tabular}




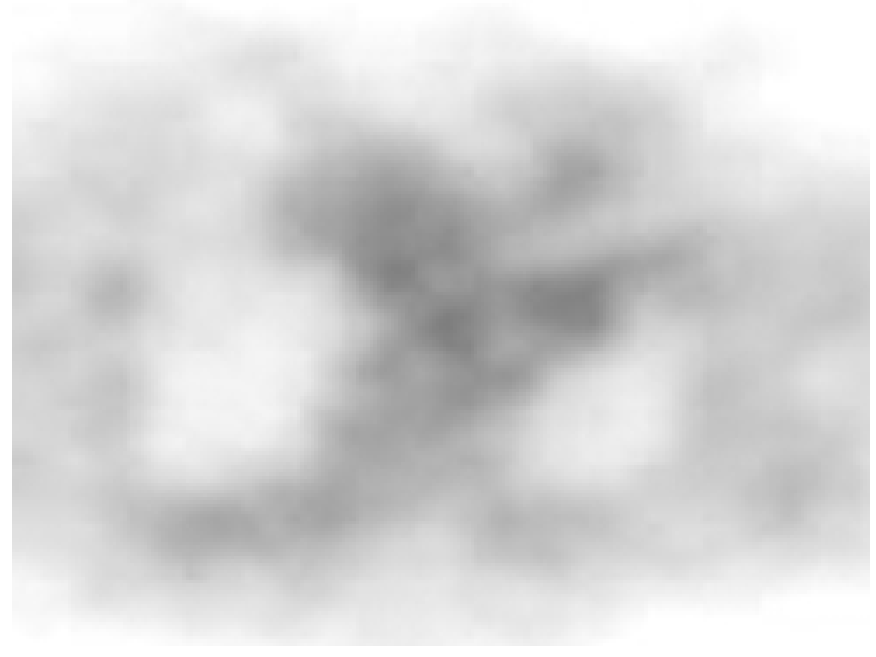

Fig. 1. Imagen transaxial de PET-FDG de NPS en LSI. Resultado indeterminado (SUV=2,8). Diagnóstico definitivo: hamartoma.

En los 14 pacientes con lesión nodular pulmonar indeterminada y antecedentes de neoplasia se obtuvo 8 casos VP, 5 VN (1 melanoma, 1 ampolla de Water, 2 de cabeza y cuello, 1 vesical) y 1 caso FN (fibrohistiocitoma maligno).

El intervalo del SUV máximo en las lesiones benignas fue de 0,4 a 2,8 (Fig. 2), con un valor medio de 1,18. Entre los nódulos pulmonares benignos de diagnóstico específico destacaron: 7 granulomas secundarios a infección por tuberculosis, 3 granulomas no infecciosos, 3 hamartomas, 1 fibrolipoma, 2 neumonías. En los 14 enfermos restantes el nódulo pulmonar permanece sin cambios bajo seguimiento clínico y radiológico

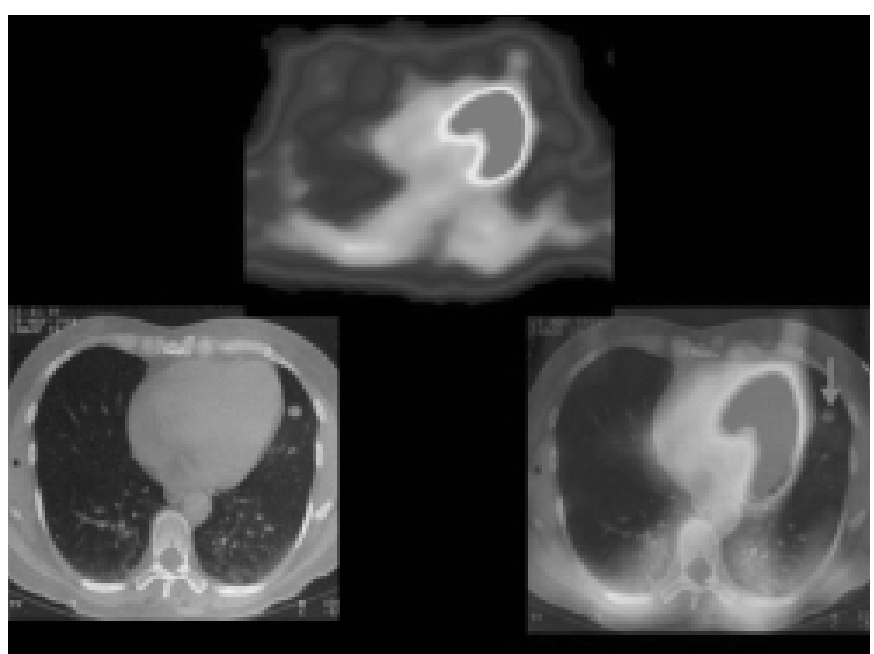

Fig. 2. Fusión TAC-PET en NPS de origen benigno en língula. Resultado VN (no fijación de FDG).

(RX cada 6 meses durante los dos primeros años), con una media actual de seguimiento de $18 \pm 6$ meses.

En las lesiones malignas el intervalo del SUV máximo fue de 0,7 a 26 (Fig. 3), con un valor medio de 7.4, diagnosticándose 28 neoplasias primarias, (13 adenocarcinomas, 12 carcinomas epidermoides, 1 carcinoma bronquioloalveolar, 1 adenocarcinoma de células pequeñas y 1 tumor carcinoide), 8

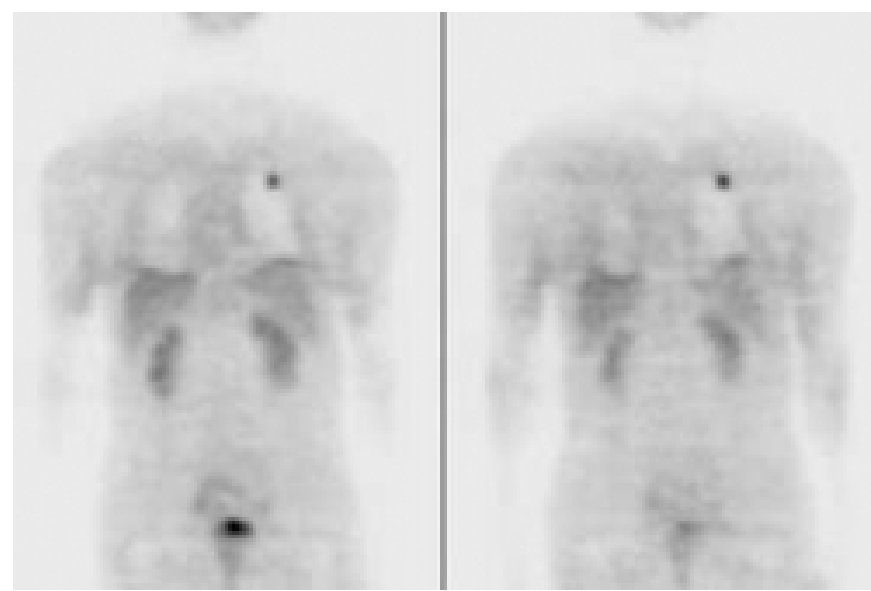

Fig. 3. Imagen coronal de PET-FDG de NPS en LSI. VP (SUV=4.4). Diagnóstico definitivo: adenocarcinoma.

lesiones metastásicas secundarias y 1 caso de supuesta malignidad sin confirmación histológica.

Si clasificamos el máximo SUV de las lesiones en tres niveles, (mayor o igual a 2, 2,5, y 3) podemos identificar (Tabla II) una mejor sensibilidad y valor predictivo negativo para el nivel de captación igual o superior a 2,5.

\begin{tabular}{|c|c|c|c|}
\hline \multicolumn{4}{|c|}{ TABLA II } \\
\hline \multicolumn{4}{|c|}{ EXACTITUD DIAGNÓSTICA DEL SUV } \\
\hline & SUV $20>$ & SUV $2,50>$ & SUV $30>$ \\
\hline S & 0,92 & 0,92 & 0,88 \\
\hline$E$ & 0,70 & 0,90 & 1,00 \\
\hline VPP & 0,79 & 0,92 & 1,00 \\
\hline VPN & 0,87 & 0,90 & 0,87 \\
\hline ED & 0,82 & 0,91 & 0,93 \\
\hline
\end{tabular}

\section{DISCUSIÓN}

Pese al uso de la RX de tórax, la TAC convencional o de alta resolución y la RMN, casi un $40 \%$ de los nódulos pulmonares solitarios (NPS) estudiados obtiene la calificación de "indeterminados". El estudio de los NPS incluye la valoración clínica, radiográfica y la realización de broncoscopia, biopsia transtorácica o incluso toracoscopia. Cuando estas maniobras no son concluyentes o están contraindicadas para los pacientes, podemos utilizar la PET-FDG como un procedimiento diagnóstico complementario y no invasivo (1).

La diferenciación de las lesiones pulmonares se puede efectuar por PET mediante análisis cualitativo o visual, o bien semicuantificando el metabolismo de glucosa tisular mediante el SUV (18). Tras efectuar la administración intravenosa de la F18-FDG, ésta se convierte en glucosa-6-fosfato mediante la hexoquinasa. Las células neoplásicas van a presentar una mayor captación de FDG y un mayor hipermetabolismo, debido a una mayor actividad de la enzima hexoquinasa, favorecida por la hipoxia y la sobre-expresión de genes codificadores de la síntesis de esta enzima, así como de proteínas transporta- 
doras de membrana para la glucosa (GLUT-1 y GLUT-3) (23).

Lowe y Naunheim (15) valoran múltiples estudios de PET-FDG para detectar lesiones pulmonares malignas. En un total de 555 pacientes, la PET-FDG obtenía una sensibilidad y especificidad de $95 \%$ y $81 \%$, respectivamente. En el mismo año, Lowe VJ y otros colaboradores (17) incluyeron en su estudio 89 pacientes con nódulo pulmonar solitario de carácter indeterminado en TAC. La sensibilidad y especificidad para todos los tamaños de las lesiones fue de $98 \%$ y $69 \%$ para el análisis visual y de $92 \%$ y $90 \%$ utilizando el SUV. Mediante el uso del criterio visual se obtenía un mayor número de falsos positivos, mejorado con el cálculo del SUV. Como desventaja, la consideración única del SUV en lesiones pequeñas $(<1,5 \mathrm{~cm})$ puede resultar en un aumento de los falsos negativos, a causa del efecto de volumen parcial.

Podemos encontrar en la PET-FDG resultados falsos negativos, sobre todo en tres situaciones: a) lesiones pequeñas que se omiten debido a la limitación de la resolución, de forma que nódulos subcentimétricos se detectan sólo si la captación de FDG es muy alta; b) determinados tipos de tumores (broncoalveolares y carcinoides) muestran menor avidez por la FDG. La menor fijación puede relacionarse con una mejor diferenciación celular y un lento crecimiento; c) la hiperglucemia puede originar una disminución de actividad en el tumor por inhibición competitiva. Los falsos positivos de la PETFDG se originan normalmente por afecciones inflamatorias, sobre todo en enfermedades granulomatosas (histoplasmosis y tuberculosis) $(9-17,20,24)$.

La PET-FDG sería de mayor utilidad en los casos, en los que el resultado de la PET influyera en el manejo del paciente, aconsejando efectuar toracotomía o bien seguimiento. Los pacientes de alto riesgo serían candidatos a la PET, previo a iniciar un procedimiento invasivo. Por el contrario en aquellos casos de alta sospecha clínica (enfermedad metastásica) o bien en los que la toracotomía no es una terapia aconsejada (masa inoperable), la PET-FDG no estaría indicada (16).

Recientemente, Gould MK y cols. (22) publican un metaanálisis de la exactitud diagnóstica de la PET-FDG en la valoración de lesiones pulmonares. Para las 1474 lesiones pulmonares focales de cualquier tamaño la máxima sensibilidad y especificidad fue de 91,2\%. Según estos autores la sensibilidad y especificidad de la PET-FDG se sitúa en un 96,8 y $77,8 \%$, respectivamente, mostrando así una alta sensibilidad y una especificidad intermedia en el diagnóstico de lesiones pulmonares malignas. Su bajo nivel de resultados falsos negativos (próximo a un 7\%) convierten a la PET-FDG en un método de elevada rentabilidad diagnóstica, ya que cuando la
PET es negativa la probabilidad de malignidad es baja, evitando la cirugía innecesaria en aquellos pacientes con NPS de baja probabilidad de malignidad.

Para Gould y cols. (26), la mejor actitud terapéutica en el manejo de los NPS, depende de la probabilidad de cáncer (pCa) que se establezca para cada paciente en función de criterios clínicos (edad del paciente, antecedentes, síntomas, tabaquismo), radiográficos y tomográficos. El análisis bayesiano es el más utilizado para calcular la pCa, de forma que si la $\mathrm{pCa}$ es baja $(<0,05)$ la mejor estrategia es observar y seguir, si la pCa es intermedia $(0,05-0,6)$ la biopsia y cuando la pCa es alta $(>0,60)$, la cirugía inmediata tras el diagnóstico. En el estudio de Dewan y cols (16) la PET-FDG demuestra ser superior al análisis tradicional Bayesiano en la clasificación correcta de benignidad-malignidad de los NPS. Además, el implementar al TAC la PET-FDG, ha demostrado ser una estrategia diagnóstica costo-efectiva básicamente por la disminución del número de cirugías (aproximadamente 15\%), permitiendo un ahorro por paciente que oscila entre los 91 \$ a los 2200 \$ (28). La estrategia TAC + PET obtuvo una mejor relación costo-efectividad cuando la probabilidad de malignidad del nódulo se situaba en 0,12 a 0,69 (28). Según Ost D y cols. (8), los pacientes con probabilidad intermedia de malignidad serían los candidatos a efectuar PET-FDG, permitiendo así una estratificación más precisa del potencial de malignidad. En los pacientes mayores con patologías médicas concomitantes el evitar un procedimiento quirúrgico es importante. Si la PET-FDG es negativa, una estrategia de seguimiento por TAC sería adecuada. Análogamente si la PET-FDG es positiva justificaría el riesgo quirúrgico. En los enfermos, sin conmorbilidad, pero con relativo alto riesgo de cáncer, efectuar una toracotomía precoz es la mejor alternativa para estos autores (8).

En la actualidad la utilización de la PET-FDG es un procedimiento diagnóstico aprobado en España por la Agencia de Evaluación de Tecnología Sanitarias (AETS), tanto en el estudio de NPS de etiología indeterminada, como en el estadiaje del carcinoma no microcítico de pulmón (23).

En nuestro trabajo hemos obtenido unos resultados similares a los publicados recientemente por Gould MK y cols. (22), con una menor sensibilidad ( $92 \%$ vs $96,8 \%$ ) y una especificidad superior ( $85 \%$ vs 77,8\%). Podemos atribuir nuestra mejor especificidad a la utilización adicional del SUV y pensamos que la diferencia de sensibilidad puede estar relacionada con el tamaño de las lesiones incluidas en cada trabajo. Según nuestros resultados, valores de SUV iguales o superiores a 2,5 aportarían la máxima sensibilidad y valor predictivo negativo al diagnóstico diferencial del NPS.

\section{Bibliografía}

1. Shulkin AN. Management of the indeterminate solitary pulmonary nodule: a pulmonologist's view. Ann Thorac Surg 1993; 56: 743-744.

2. Fletcher JW. PET scanning and the solitary pulmonary nodule. Semin Thorac Cardiovasc Surg 2002; 14: 268-274.

3. Berger WG, Erly WK, Krupinski EA, Standen JR, Stern RG. The solitary pulmonary nodule on chest radiography: can we really tell if the nodule is calcified? AJR Am J Roentgenol 2001; 176: 201-204.

4. Siegelman SS, Khouri NF, Leo FP, Fishman EK, Braverman RM, Zerhouni EA. Solitary pulmonary nodules: CT assessment. Radiology 1986; 160: 307-312.
5. Seeman MD, Staebler A, Beinert T, Dienemann H, Obst B, Matzko M et al. Usefulness of morphological characteristics for the differentiation of benign from malignant solitary pulmonary lesions using HRCT. Eur Radiol 1999; 9: 409-417.

6. Peng G, Cai Z, Gao Y. The value of $\mathrm{CT}$ and MRI in differentiating malignant nodule from tuberculoma. Zhonghua Jie He Hu Xi Za Zhi 1995; 18: 218-220.

7. Khouri NF, Meziane MA, Zerhouni EA, Fishman EK, Siegelman SS The solitary pulmonary nodule: Assessment, diagnosis and management. Chest 1987; 91: 128-133. 
8. Ost D, Fein A. Evaluation and management of the solitary pulmonary nodule. Am J Respir Crit Care Med 2000; 126: 782-787.

9. Kubota K, Matsuzawa T, Fujiwara T, Ito M, Hatazawa J, Ishiwata K et al. Differential diagnosis of lung tumor with positron emission tomography: A prospective study. J Nucl Med 1990; 31: 1927-1932.

10. Patz EF Jr, Lowe VJ, Hoffman JM, Paine SS, Burrowers P, Coleman RE et al. Focal pulmonary abnormalities: Evaluation with F-18 fluorodeoxyglucose PET scanning. Radiology 1993; 188: 487-490.

11. Bury T, Dowlati A, Paulus P, Corhay JL, Benoit T, Kayembe JM et al. Evaluation of the solitary pulmonary nodule by positron emission tomography imaging. Eur Respir J 1996; 9: 410-414.

12. Knight SB, Delbeke D, Stewart JR, Sandler MP. Evaluation of pulmonary lesions with FDG-PET comparison of findings in patients with and without a history of prior malignancy. Chest 1996; 109: 982988.

13. Gupta NC, Maloof J, Gunel E: Probability of malignancy in solitary pulmonary nodules using Fluorine-18-FDG and PET. J Nucl Med 1996; 37: 943-948.

14. Lowe VJ, Duhaylongsod FG, Patz EF, Delong DM, Hoffman JM, Wolfe WG et al. Pulmonary abnormalities and PET data analysis: A retrospective study. Radiology 1997; 202: 435-439.

15. Lowe VJ, Naunheim KS. Positron emission tomography in lung cancer. Ann Thorac Surg 1998; 65: 1821-1829.

16. Dewan NA, Shehan CJ, Reeb SD, Gobar LS, Scott WJ, Ryschon K Likelihood of malignancy in a solitary pulmonary nodule comparison of Bayesian analysis and results of FDG-PET scan. Chest 1997; 112: 416422.

17. Lowe VJ, Fletcher JW, Gobar L, Lawson M, Kirchner P, Valk P et al. Prospective investigation of positron emission tomograhy in lung nodules. J Clin Oncol 1998; 16: 1075-1084.
18. Huang SC. Anatomy of SUV. Standardized uptake value. Nucl Med Biol 2000; 27: 643- 646.

19. Agencia de Evaluación de Tecnologías Sanitarias (AETS) Instituto de Salud Carlos III- Ministerio de Sanidad y Consumo. Tomografía por Emisión de Positrones (PET) con 18FDG en Oncología Clínica (Revisión Sistemática). Madrid: AETS- Instituto de Salud Carlos III, Noviembre de 2001.

20. Coleman RE. PET in lung cancer. J Nucl Med 1999; 40: 814-820.

21. Hung GU, Shiau YC, Tsai SC, Ho YJ, Kao CH, Yen RF. Differentiation of radiographically indeterminate solitary pulmonary nodules with FDG PET. Jpn J Clin Oncol 2001; 31: 51-54.

22. Gould MK, Maclean CC, Kuschner WG, Rydzak CE, Owens DK. Accuracy of positron emission tomography for diagnosis of pulmonary nodules and mass lesions: a meta-analysis. JAMA 2001; 285: 914-924.

23. Protocolo de Uso Tutelado para la recogida de Información sobre la Utilización de la 18FDG-PET. Documento de Consenso. Madrid, mayo del 2002. Agencia de Evaluación de Tecnologías Sanitarias. Instituto de Salud Carlos III. Ministerio de Sanidad y Consumo.

24. Carreras JL, Lapeña L, Asensio C, eds. PET en Oncología. Madrid: Nova Sidonia Oncología-Hematología, 2002.

25. Carreras Delgado JL, Asensio del Barrio C, Lapeña Gutiérrez L. La tomografía por emisión de positrones en el estudio del nódulo pulmonar solitario. Rev Clin Esp 2000; 7: 382-384.

26. Gould MK, Lillington GA. Strategy and cost in investigating solitary pulmonary nodules. Thorax 1998; 53: S32-S37.

27. Lillington GA, Cummings SR. Decision analysis approaches in solitary pulmonary nodules. Semin Respir Med 1989; 10: 227-231.

28. Gambhir SS, Shepherd JE, Shah BD, Hart E, Hoh CK, Valk PE y cols. Analytical decision model for the cost-effective management of solitary pulmonary nodules. J Clin Oncol 1998; 16: 2113-2125. 